\title{
Cross-linked main-chain polybenzoxazine nanofibers by photo and thermal curing; stable at high temperatures and harsh acidic conditions
}

\author{
Yelda Ertas ${ }^{\text {a, b }}$, Tamer Uyar ${ }^{\text {a, b, * }}$ \\ ${ }^{a}$ Institute of Materials Science \& Nanotechnology, Bilkent University, Ankara, 06800, Turkey \\ ${ }^{\mathrm{b}}$ UNAM-National Nanotechnology Research Center, Bilkent University, Ankara, 06800, Turkey
}

\section{A R T I C L E I N F O}

\section{Article history:}

Received 28 July 2015

Received in revised form

11 December 2015

Accepted 17 December 2015

Available online 21 December 2015

\section{Keywords:}

Main-chain polybenzoxazine

Electrospinning

Nanofibers

Cross-linked

Photo curing

Thermal curing

\begin{abstract}
A B S T R A C T
In this study, for the first time cross-linking of linear aliphatic diamine-based main-chain polybenzoxazine ( $\mathrm{MCPBz}$ ) electrospun nanofibers were accomplished by two-step approach consisting of photo and thermal curing. Initially, two novel MCPBz resins which comprise of a benzophenone unit in the polymer main-chain were synthesized and uniform MCPBz nanofibers were produced by electrospinning. At first step, photo curing was performed by free radical polymerization initiated by UV-light and thermal stability of nanofibers was enhanced. At second step, thermal curing was carried out at different temperatures $\left(150-225^{\circ} \mathrm{C}\right)$ and ring opening and cross-linking of benzoxazine groups in the fiber structure were achieved. After two-step curing, cross-linked MCPBz nanofibers were obtained as free-standing material with good mechanical properties. Moreover, it was shown that these two crosslinked MCPBz nanofibers were structurally stable and maintained their fibrous morphology at high temperatures $\left(400{ }^{\circ} \mathrm{C}\right.$ ), in good solvents (chloroform, DMF, 1,4-dioxane, DMAc, THF) and highly concentrated strong acids $\left(\mathrm{HCl}, \mathrm{HNO}_{3}, \mathrm{H}_{2} \mathrm{SO}_{4}\right)$.
\end{abstract}

() 2015 Elsevier Ltd. All rights reserved.

\section{Introduction}

Polybenzoxazines as a new member of thermosetting phenolic resins are of great interest for various scientific and industrial fields owing to their superior physical and thermal properties. Meanwhile, their wide range of molecular design flexibility which enables the tailoring the properties of the final product for specific applications make them much more attractive in different application areas including electronic packaging, composites, high performance adhesives, non-flammable materials, transportation and aerospace industries [1].

Basically, benzoxazines are synthesized from mono-functional phenols and amines with different substitutional groups. Besides, any combination of a difunctional phenol and primary amine and in a similar manner, diamine and mono-functional phenol can be used for the synthesis of benzoxazines [1]. However, a more recent concept of benzoxazine resins involves the use of a difunctional

\footnotetext{
* Corresponding author. Institute of Materials Science \& Nanotechnology, Bilkent University, Ankara, 06800, Turkey.

E-mail address: uyar@unam.bilkent.edu.tr (T. Uyar).
}

phenolic derivative and a diamine, producing a linear polymer having oxazine rings in the main-chain called as main-chain polybenzoxazine (MCPBz) [1]. This type of benzoxazines can be quite useful for the production of nanofibers from this kind of materials since chain entanglement and overlapping is the key factor for the production of nanofibers by electrospinning. Electrospinning is a widely used technique to produce multifunctional nanofibers from remarkable range of organic and inorganic materials including polymers, polymer blends, composites, sol-gels, ceramics, etc [2,3]. Nanofibers produced by this technique have a very large surface area to volume ratio and nanoscale pores having distinctive chemical, physical and mechanical properties when compared to their bulk or film forms. Polybenzoxazines are extensively studied in the literature with their different forms such as bulk [4-8], film [9-19], aerogel [20-25], porous membrane [26,27], etc. for various applications. On the other hand, electrospinning of nanofibers from polybenzoxazine resins is a new concept and electrospinning of MCPBz resins without using additional polymeric matrices was first reported recently by our group [28].

Generally, polymerization/cross-linking of benzoxazines can be achieved by thermal curing which is a thermally induced ring opening reaction of benzoxazines and $\mathrm{MCPBz}$ occur at around 
$200{ }^{\circ} \mathrm{C}[1]$. First successful study for the cross-linking of $\mathrm{MCPBz}$ nanofibers by thermal curing was reported by Li et al. [29]. They synthesized the MCPBz by using 4,4'-diaminophenylether, bisphenol-A, and formaldehyde as starting materials and they produced $\mathrm{MCPBz}$ nanofibers which are mechanically robust and stable under harsh environmental conditions. Since the melting point of the nanofibers were higher than their curing temperatures, they achieved to obtain cross-linked $\mathrm{MCPBz}$ nanofibers by thermal curing. However, in our previous study we could not able to produce cross-linked nanofibers from long linear aliphatic diaminebased $\mathrm{MCPBz}$ nanofibers by directly thermal curing because of the very low melting points of PBA-ad6 and PBA-ad12 nanofibers (73 and $42{ }^{\circ} \mathrm{C}$ ) which are quite lower than their curing temperatures $\left(203\right.$ and $\left.205^{\circ} \mathrm{C}\right)$. Therefore, in this study we focus on twostep curing procedure including the photo and thermal curing for the cross-linking of this type of $\mathrm{MCPBz}$ nanofibers. Although, thermal curing is very common and basic method to polymerize or crosslink benzoxazines and MCPBzs, there are few studies in literature that use photo curing for the polymerization of benzoxazines $[30,31]$. In addition, benzophenone based benzoxazine monomers were synthesized and used as photoinitiator for the photopolymerization of acrylate monomers [32-35]. Besides, both photopolymerization as a preliminary step and thermally activated polymerization for the ring-opening and cross-linking of methacryloyl functional benzoxazines were studied [36]. All these research works provide us useful information on designing new kind of benzoxazine resins to improve the curing procedure of $\mathrm{MCPBz}$ nanofibers in order to achieve cross-linking without deteriorating the fiber structure.

For this purpose, initially, two novel MCPBz (DHBP-ad6 and DHBP-ad12) which consist of a benzophenone unit in the polymer main-chain were synthesized. Due to the presence of benzophenone unit in the main-chain, DHBP-ad6 and DHBP-ad12 nanofibers are able to crosslink by UV-light initiated free radical polymerization. Therefore, by synthesizing benzophenone containing $\mathrm{MCPBz}$, we aimed to provide preliminary cross-linking through photo curing to enhance the thermal stability of nanofibers for thermal curing in which ring-opening and almost complete cross-linking can be achieved as maintaining the nanofibrous structure.

\section{Experimental}

\subsection{Materials}

Paraformaldehyde (Sigma-Aldrich, 95\%), 1,4dihydroxybenzophenone (DHBP, Alfa-easer, 98\%), 1,12diaminododecane (Aldrich, 98\%) and 1,6-diaminohexane (Aldrich, 98\%) were used without further purification. Chloroform (Sigma-Aldrich, 99\%), N,N-dimethylformamide (DMF, Fluka, 98\%), tetrahydrofuran (THF, Merck, 99.7\%) 1,4-dioxane (Sigma-Aldrich, 99\%), dimethylacetamide (DMAc, Sigma-Aldrich, 99\%), hydrochloric acid ( $\mathrm{HCl}$, Sigma-Riedel, 37\%), sulfuric acid $\left(\mathrm{H}_{2} \mathrm{SO}_{4}\right.$, Sigma-Riedel, 95\%), nitric acid $\left(\mathrm{HNO}_{3}\right.$, Sigma-Riedel, 65\%) and methanol (Sigma-Aldrich, 99.7\%) were used as received. FTIR grade potassium bromide (Sigma-Aldrich, 99\%) and deuterated chloroform (Merck, 99.8\%) were also used as received.

\subsection{Synthesis of main-chain polybenzoxazine (MCPBz) resins; DHBP-ad6 and DHBP-ad12}

Two novel MCPBz were synthesized by using two different amines (1, 6-diaminohexane and 1,12-diaminododecane), phenolic derivative (1,4-dihydroxybenzophenone) and paraformaldehyde as starting materials. For the synthesis of first MCPBz named as DHBPad6, 1,6-diaminohexane (10 mmol), 1,4-dihydroxybenzophenone
(10 mmol) and paraformaldehyde $(40 \mathrm{mmol})$ were dissolved in $200 \mathrm{ml}$ chloroform and refluxed at $60^{\circ} \mathrm{C}$ for $30 \mathrm{~h}$. For the synthesis of second MCPBz named as DHBP-ad12, 1,12-diaminododecane (10 mmol), 1,4-dihydroxybenzophenone $(10 \mathrm{mmol})$ and paraformaldehyde ( $40 \mathrm{mmol}$ ) were dissolved in $200 \mathrm{ml}$ chloroform and refluxed at $60^{\circ} \mathrm{C}$ for $36 \mathrm{~h}$. Reaction mixtures were kept in the fume hood to cool down at room temperature, then chloroform was evaporated completely from the reaction mixture by using rotaryevaporator system and the product were dried under vacuum at $35^{\circ} \mathrm{C}$ for $24 \mathrm{~h}$. In order to remove any residual reactants, DHBP-ad6 was purified by washing through with cold methanol several times and then dried over night under vacuum at $35{ }^{\circ} \mathrm{C}$. Overall yield of the synthesized DHBP-ad6 and DHBP-ad12 were $76 \%$ and $87 \%$, respectively. According to the GPC measurements, weight average molecular weight (Mw) and polydispersity index (PDI) of DHBPad6 and DHBP-ad12 were calculated as $~ 10,000$ (PDI:2.5) and $\sim 15,000$ (PDI:2.8), respectively.

\subsection{Electrospinning of DHBP-ad6 and DHBP-ad12 nanofibers}

The homogenous solutions of DHBP-ad6 and DHBP-ad12 were prepared in chloroform/DMF mixture solvent system (chloroform:DMF; 4:1, v/v) and solution concentrations were varied from $20 \%$ to $35 \%(\mathrm{w} / \mathrm{v})$ and from $15 \%$ to $25 \%(\mathrm{w} / \mathrm{v})$, respectively. The solutions were taken in $3 \mathrm{ml}$ syringes with metallic needle of $0.4 \mathrm{~mm}$ outer diameter and the syringe was positioned horizontally on the syringe pump (KD Scientific, KDS 101). Then the positive electrode of the high voltage power supply (Spellman, SL60) was clamped to the metal needle. Metal plate collector covered by a piece of aluminum foil was placed across the horizontally positioned syringe to collect nanofibers. In order to optimize the electrospinning parameters, flow rate of the polymer solution $(0.5-1.5 \mathrm{ml} / \mathrm{h})$, applied voltage $(10-20 \mathrm{kV})$ and tip-to-collector distance $(10-20 \mathrm{~cm})$ were varied within the ranges given in the parenthesis. For both of the $\mathrm{MCPBz}$, the most favorable results were obtained when the flow rate of the polymer solution was $0.75 \mathrm{ml} / \mathrm{h}$, applied voltage was $15 \mathrm{kV}$ and tip-to-collector distance was $10 \mathrm{~cm}$. Electrospinning was performed in the completely enclosed plexiglas box and the inside temperature and relative humidity were $22{ }^{\circ} \mathrm{C}$ and $18 \%$, respectively. After the electrospinning, the collected nanofibers were dried over night at room temperature inside the fume hood in order to remove any residual solvent.

\subsection{Curing studies of DHBP-ad6 and DHBP-ad12 nanofibers}

Two-step curing (photo and thermal) procedure was applied to DHBP-ad6 and DHBP-ad12 nanofibers to obtain cross-linked polybenzoxazine nanofibers. At first step, a piece of sample $(\sim 2 \times 5 \mathrm{~cm})$ from each nanofibers were put under the UV lamp (Osram Ultravitalux, $300 \mathrm{~W}$ ) from different distances $(7,10$ and $15 \mathrm{~cm}$ ) and various irradiation times ( $15 \mathrm{~min}-12 \mathrm{~h}$ ) in order to determine optimum parameters for the photo curing. For the DHBP-ad6 nanofibers, curing time and distance between lamp and the sample were determined to be $1 \mathrm{~h}$ and $10 \mathrm{~cm}$, respectively. On the other hand, for the DHBP-ad12 nanofibrous, these parameters determined to be $1 \mathrm{~h}$ and $15 \mathrm{~cm}$, respectively. At second step, photocured samples were put in the standard oven and kept for $1 \mathrm{~h}$ at different temperatures $\left(150{ }^{\circ} \mathrm{C}, 175^{\circ} \mathrm{C}, 200^{\circ} \mathrm{C}\right.$ and $\left.225^{\circ} \mathrm{C}\right)$ to provide opening and cross-linking of the oxazine ring in the structure of nanofibers with thermal curing. Small amount of sample was taken from each $\mathrm{MCPBz}$ nanofibers at different temperatures to investigate the ring-opening reaction and cross-linking by FTIR. 


\subsection{Solubility and stability test of cross-linked DHBP-ad6 and DHBP-ad12 nanofibers}

Solubility of the cross-linked DHBP-ad6 and DHBP-ad12 nanofibers after photo curing and after thermal curing were tested with some good solvents; chloroform, N,N-dimethylformamide (DMF), 1,4-dioxane, dimethylacetamide (DMAc) and tetrahydrofuran (THF). In addition, stability of these cross-linked nanofibers in harsh conditions were evaluated by strong acids; $\mathrm{HCl}, \mathrm{HNO}_{3}, \mathrm{H}_{2} \mathrm{SO}_{4}$. $5 \mathrm{M}$ aqueous solutions were prepared from each type of acid and $5 \mathrm{ml}$ of acid solutions were poured into vials. Likewise, $5 \mathrm{ml}$ of solvent was poured into vials and a piece of sample with the dimensions of around $1 \times 1 \mathrm{~cm}$ from each photo and thermal-cured DHBP-ad6 and DHBP-ad12 nanofibers were placed into vials separately. Samples were kept in solvents and acid solutions for $24 \mathrm{~h}$ and then dried at room temperature in the fume hood over night. In addition, thermal stability of cross-linked DHBP-ad6 and DHBP-ad12 nanofibers were investigated by keeping nanofibers at different temperatures $\left(250^{\circ} \mathrm{C}, 275^{\circ} \mathrm{C}, 300^{\circ} \mathrm{C}, 350^{\circ} \mathrm{C}\right.$ and $\left.400{ }^{\circ} \mathrm{C}\right)$.

\subsection{Measurements and characterization}

The structure of the synthesized DHBP-ad6 and DHBP-ad12 were confirmed by proton nuclear magnetic resonance (1H NMR, Bruker Advance III $400 \mathrm{MHz}$ ) spectrometer. Samples were prepared by dissolving about $20 \mathrm{mg} / \mathrm{ml}$ polybenzoxazines in deuterated chloroform $\left(\mathrm{CDCl}_{3}\right)$. Fourier transform infrared (FTIR, BrukerVERTEX70) spectrometer was employed to verify the structure of the synthesized $\mathrm{MCPBz}$ resins and also confirm the ring-opening reaction of DHBP-ad6 and DHBP-ad12 during the curing process. FTIR spectra were obtained with 64 scans at a resolution of $4 \mathrm{~cm}^{-1}$ within $4000-400 \mathrm{~cm}^{-1}$ range. Samples were prepared by grinding with $\mathrm{KBr}$ in a ratio around 3:100 (3 mg sample: $100 \mathrm{mg} \mathrm{KBr}$ ) and then compressed to form discs. UV-visible spectrophotometer (Varian Cary-100) was used to confirm the existence of the photo active group in the structure of synthesized MCPBz. Samples were dissolved in chloroform and clear solutions were placed into quartz cuvettes for the UV-Vis measurements. Scanning electron microscope (SEM, Quanta 200 FEG, FEI) was used to investigate the morphology and the diameter distribution of nanofibers. Samples were coated with $5 \mathrm{~nm} \mathrm{Au} / \mathrm{Pd}$ (PECS-682) prior to the SEM imaging and the average fiber diameter (AFD) was calculated by analyzing around 100 fibers from the SEM images. Differential scanning calorimetry (DSC, TA Instruments Q20) experiments were conducted to study thermal transitions of $\mathrm{MCPBz}$ nanofibers under nitrogen atmosphere at a heating rate of $10{ }^{\circ} \mathrm{C} / \mathrm{min}$. Thermal properties of the nanofibers were investigated with thermogravimetric analyzer (TGA, Q500, TA Instruments) by starting from room temperature to $800^{\circ} \mathrm{C}$ under nitrogen gas at a heating rate of $20^{\circ} \mathrm{C} /$ min. Molecular weight and molecular weight distribution of the PBA-ad6 and PBA-ad12 were determined by gel permeation chromatography (GPC, Agilent Techn. 1200 Series) equipped with Zorbax PSM 300-S columns and ELSD detector. THF used as the mobile phase at a flow rate of $1 \mathrm{~mL} / \mathrm{min}$. Samples were dissolved in the THF at approximately $1 \mathrm{mg} / \mathrm{ml}$. Calibration of the system was performed with polystyrene standards having molecular weight of $500-1 \times 10^{6} \mathrm{~g} / \mathrm{mol}$. Mechanical properties of the cross-linked nanofibers were measured by using DMA (Q800 TA Instruments) equipped with tensile fixture. Rectangular shaped samples were prepared with dimensions of around $15 \times 2.0 \times 0.03 \mathrm{~mm}$ for DHBPad12 and $15 \times 2.0 \times 0.04 \mathrm{~mm}$ for DHBP-ad12. Stress-strain curve of the three replicate from each type of $\mathrm{MCPBz}$ nanofibers were obtained at $0.025 \mathrm{~N} / \mathrm{min}$ force ramp.

\section{Results and discussion}

\subsection{Structural characterization of DHBP-ad6 and DHBP-ad12 resins}

DHBP-ad6 and DHBP-ad12 were synthesized as two novel $\mathrm{MCPBz}$ by using difunctional amines (1,6-diaminohexane and 1,12diaminododecane), difunctional phenol (1,4dihydroxybenzophenone) (DHBP) and paraformaldehyde as starting materials. Since there are two hydroxyl groups on DHBP and two amino groups on both 1,6-diaminohexane and 1,12diaminododecane, two oxazine rings are formed in one repeating unit of each MCPBz as shown in Scheme 1. The synthesized polybenzoxazines are main-chain type polybenzoxazine (MCPBz) consisting of repeating units of bifunctional benzoxazine structure bonded to different chain length of aliphatic diamine to form the macromolecular chain which possess benzophenone and coinitiator amine in the main-chain. Formation of this structure is the evidence of successful synthesis of the benzoxazines and this structure of the synthesized DHBP-ad6 and DHBP-ad12 were confirmed by ${ }^{1} \mathrm{H}$ NMR, FTIR and UV-Vis spectroscopies.

The proposed chemical structures and ${ }^{1} \mathrm{H}$ NMR spectra of DHBPad6 and DHBP-ad12 are given in Fig. S1. For both MCPBz, the characteristic benzoxazine resonance bands corresponding to the methylene units of oxazine ring; $\mathrm{O}-\mathrm{CH}_{2}-\mathrm{N}$ and the $\mathrm{Ph}-\mathrm{CH}_{2}-\mathrm{N}$ were observed at 4.04 and $4.95 \mathrm{ppm}$, respectively. Resonance bands of aliphatic protons were observed at 1.37 and $2.74 \mathrm{ppm}$ for DHBPad6, and 1.28 and $2.74 \mathrm{ppm}$ for DHBP-ad12. In addition, resonance bands of aromatic protons were observed at 6.80-7.52 ppm region as multiplet for both of the MCPBz resin. The existence of these characteristic bands in the ${ }^{1} \mathrm{H}$ NMR spectra of the both MCPBz was the evidence for the synthesis of desired benzoxazine structures.

Fig. S2 represents the FTIR spectra of the DHBP-ad6 and DHBPad12 resins. Characteristic absorption bands of the benzoxazine structure of the both $\mathrm{MCPBz}$ were observed at the same wavenumbers with different intensities. Absorption band existing at $918 \mathrm{~cm}^{-1}$ due to the out-of-plane $\mathrm{C}-\mathrm{H}$ vibration mode of the benzene ring attached to oxazine ring. Sharp absorption band observed at $1231 \mathrm{~cm}^{-1}$ due to the asymmetric stretching of $\mathrm{C}-\mathrm{O}-\mathrm{C}$ in the oxazine ring. In addition, the very intense and sharp band existing at $1496 \mathrm{~cm}^{-1}$ is due to the in-plane $\mathrm{C}=\mathrm{H}$ bending mode of the tri-substituted benzene ring. FTIR results are good agreement with the ${ }^{1} \mathrm{H}$ NMR data confirming the synthesis of two novel MCPBz resins was achieved.

Moreover, further characterization on the structure of these resins was carried out with UV-Vis spectrophotometer. Since these novel MCPBzs including benzophenone unit as a part of mainchain, it is expected to observe UV absorption for both MCPBz. UV-Vis spectra of DHBP-ad6 and DHBP-ad12 are given in Fig. S3. The main benzenoid $\pi-\pi^{*}$ type transitions of BP exist in the region of 250-300 nm [37], however, significantly red-shifted maximal absorption band observed for the synthesized novel $\mathrm{MCPBz}$ (Fig. S3) indicating the successful formation of oxazine rings. In brief, FTIR, ${ }^{1} \mathrm{H}$ NMR and UV-Vis spectroscopy analysis results show that benzophenone based MCPBz resines were successfully synthesized with the desired structure.

\subsection{Electrospinning of DHBP-ad6 and DHBP-ad12 nanofibers}

As it is known, morphological properties of the electrospun nanofibers are strongly depends on the polymer type, molecular weight of the polymer, solvent, concentration, surface tension and the conductivity of the polymer solutions [2,3]. For the polymers with very similar chemical structure as DHBP-ad6 and DHBP-ad12 resins, molecular weight is the main factor that effects the concentration of the polymer solution, hence, molecular weight of 


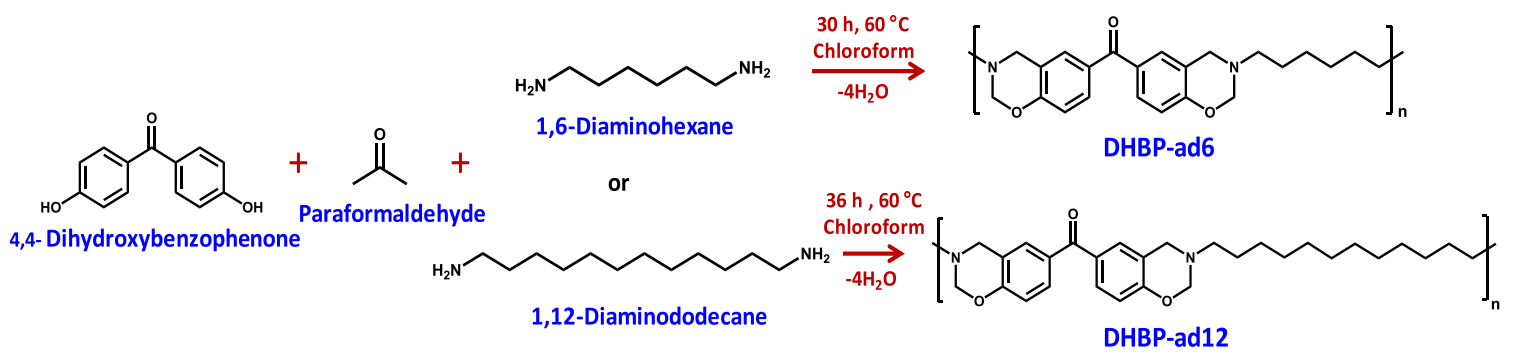

Scheme 1. Synthesis mechanisms of the DHBP-ad6 and DHBP-ad12 resins.

these resins plays a vital role on their electrospinning ability. Here, the only difference between DHBP-ad6 and DHBP-ad12 resins is the chain length of the aliphatic diamine used as a precursor for the synthesis of these resins. As abbreviated, DHBP-ad6 contains 6-C aliphatic diamine and DHBP-ad12 contains 12-C aliphatic diamine, and their molecular weights were measured by GPC as $\sim 10,000$ and $\sim 15,000 \mathrm{~g} / \mathrm{mol}$, respectively. Therefore, measured molecular weight of the DHBP-ad6 and DHBP-ad12 resins were taken into consideration while preparing the electrospinning solutions. Before determining the concentration range for the electrospinning solutions, suitable solvents system have been chosen as chloroform/DMF $(4: 1, \mathrm{v} / \mathrm{v})$ in which both MCPBz resins have formed homogenous and clear solutions. Then, different concentrations of the DHBP-ad6 and DHBP-ad12 solutions were prepared in order to produce bead-free and uniform nanofibers and here, the concentration range was given as $25-35 \%(w / v)$ for DHBP-ad6 and $15-25 \%$ $(\mathrm{w} / \mathrm{v})$ for DHBP-ad12 in which the significant morphological changes were observed. Accordingly, the morphological characteristics of the electrospun nanofibers are summarized in Table S1.

SEM images of the nanofibers electrospun from $25 \%, 30 \%$, and $35 \%(w / v)$ DHBP-ad6 solutions are shown in Fig. 1, a-c. Electrospinning of $25 \%(\mathrm{w} / \mathrm{v})$ DHBP-ad6 solution resulted beaded structure with ultrafine fibers having average fiber diameter (AFD) of $220 \pm 105 \mathrm{~nm}$ (Fig. 1a). At low solution viscosity, fewer chain entanglements and higher amount of solvent have dominant effect on the electrospinning jet resulting the formation of beads along with the fibers [2,3]. As the polymer concentration increased to $30 \%(\mathrm{w} /$ v), the number of beads decreased dramatically and elongated beaded structures with nanofibers having AFD of $440 \pm 130 \mathrm{~nm}$ were produced (Fig. 1b). When the polymer concentration reached to $35 \%(\mathrm{w} / \mathrm{v})$ in solution, bead free nanofibers having AFD of $605 \pm 145 \mathrm{~nm}$ were obtained due to the higher polymer chain entanglements in the solution provided electrospinning jet to be fully stretched for uniform fiber formation (Fig. 1c) [2,3]. SEM images of the nanofibers electrospun from $15 \%, 20 \%$, and $25 \%(\mathrm{w} / \mathrm{v})$ DHBP-ad12 solutions are given in Fig. 1, d-f. As it is observed from the SEM images, $15 \%(\mathrm{w} / \mathrm{v})$ DHBP-ad12 solution yielded beaded nanofibers having AFD of $225 \pm 90 \mathrm{~nm}$ because of the low viscosity of the polymer solution (Fig. 1d). Bead-free and uniform nanofibers were obtained when the DHBP-ad12 solution concentration was at $20 \%(w / v)$. AFD of the nanofibers electrospun from $20 \%(w / v)$ DHBPad12 solution was $380 \pm 115 \mathrm{~nm}$ (Fig. 1e). It is a typical behavior of polymeric systems in the electrospinning process that beaded nanofibers transform to bead-free fibers when the concentration and/or viscosity of the polymer solution is optimized [38]. As the concentration of the DHBP-ad12 solution increased to $25 \%(\mathrm{w} / \mathrm{v})$, the fiber diameter became thicker (AFD $=620 \pm 160 \mathrm{~nm}$ ) because of the high solution viscosity (Fig. 1, f).

Remarkably, with the increase in viscosity/concentration, the diameter of the electrospun fiber also increases. The reason for increase in fiber diameter is the greater resistance of the solution to be stretched because of the more chain entanglements at higher polymer concentration [2]. Furthermore, it is clearly observed that the aliphatic diamine chain length, accordingly the molecular
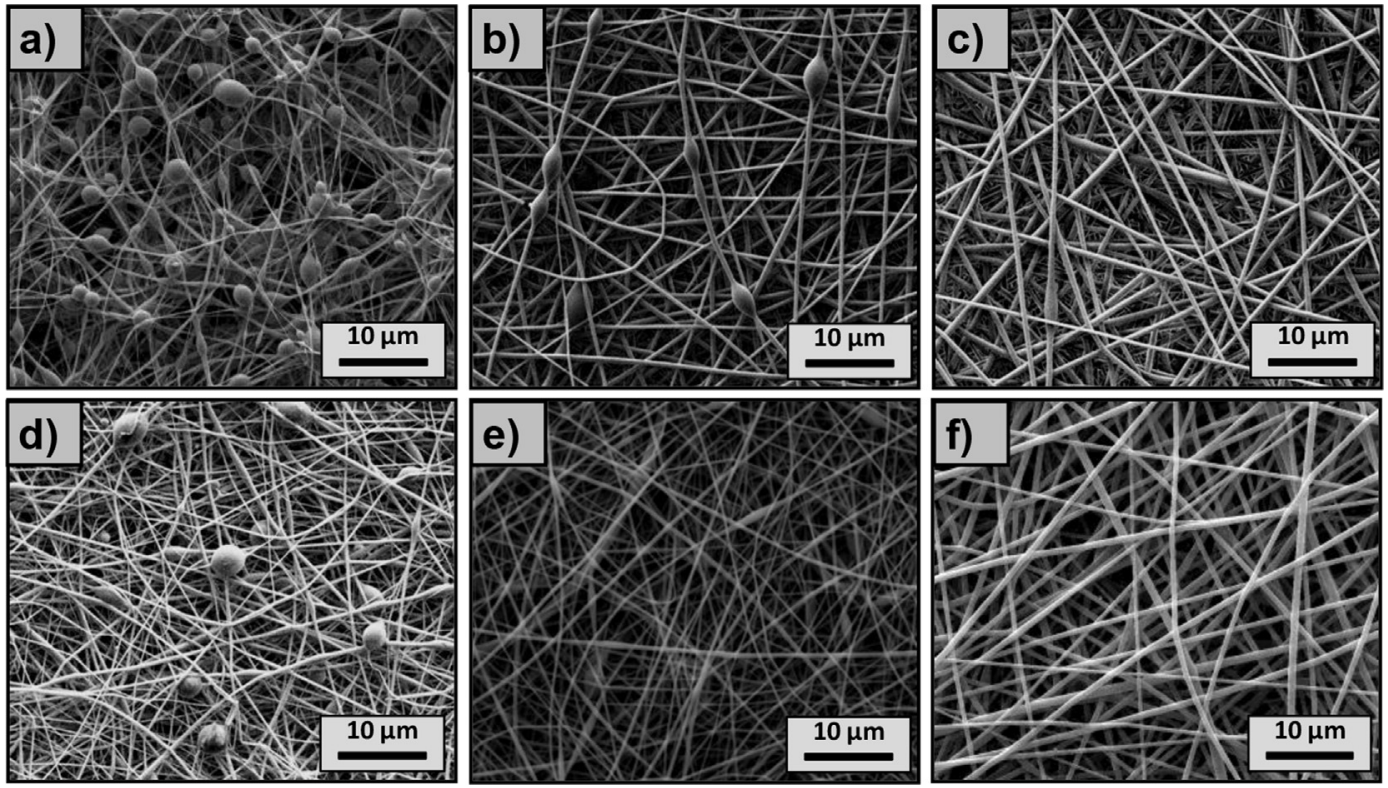

Fig. 1. Representative SEM images of the electrospun nanofibers obtained from solutions of DHBP-ad6 a) $25 \%$, b) $30 \%$, c) $35 \%$ and DHBP-ad 12 d) $15 \%$, e) $20 \%$, f) $25 \%$. 
weight of the MCPBz resins play an important role on their electrospinning ability. Presumably, higher molecular weight and longer aliphatic chain of the DHBP-ad12 resulted in more chain entanglement and overlapping in the polymer solution, thus, we achieved to obtain bead-free and uniform nanofibers at lower solution concentration for DHBP-ad12 (25\% w/v) compared with the DHBP-ad6 (35\% w/v).

\subsection{Curing studies of DHBP-ad6 and DHBP-ad12 nanofibers}

Generally, cross-linking and polymerization of the benzoxazines achieved by thermally initiated ring-opening reactions. First example of the cross-linking of polybenzoxazine-based nanofibers by directly thermal curing was given by Li and co-workers [29]. Initially, they produced nanofibers from MCPBz synthesized by using 4,4'-diaminophenylether and bisphenol-A, and then they were able to obtain cross-linked MCPBz nanofibers by thermal curing at $240{ }^{\circ} \mathrm{C}$ for $3 \mathrm{~h}$. On the contrary, our previous study demonstrated that nanofibers produced by MCPBz which based on the bisphenol-A and diamines with long aliphatic chains (1,12diaminododecane and 1,6-diaminohexane) have not shown resistance to the heat treatment during the thermal curing and nanofibers lost the fiber morphology at low temperatures around 75-100 ${ }^{\circ} \mathrm{C}$ [28]. Presumably, 4,4'-diaminophenylether provided more rigid structure to the MCPBz nanofibers compared with 1,12diaminododecane and 1,6-diaminohexane, hence, the melting temperature of the nanofibers obtained from the former one is much higher than the others. Therefore, they achieved to preserve fiber structure during the thermal treatment and obtained crosslinked nanofibers by thermal curing. Here, in order to improve the curing process of $\mathrm{MCPBz}$ nanofibers obtained from long aliphatic diamine-based polybenzoxazines, structure of the MCPBz tailored to be able to crosslink by photo curing and DHBP-ad6 and DHBP-ad12 resins which consist of a benzophenone unit in the polymer main-chain were synthesized. Initially, DSC experiments were performed to measure the thermal transition temperatures of the nanofibers obtained from these two MCPBz resins. As it is expected, melting transition was observed for DHBP-ad6 and DHBPad12 nanofibers at low temperatures as $73{ }^{\circ} \mathrm{C}$ and $54{ }^{\circ} \mathrm{C}$, respectively (Fig. 2). Nonetheless, these nanofibers were directly thermalcured and not surprisingly DHBP-ad6 and DHBP-ad12 nanofibers lost the fiber morphology by forming film even at first step of the thermal curing $\left(150{ }^{\circ} \mathrm{C}\right.$ ) (Fig. 3, b, f). In addition, exothermic peak centered at $217{ }^{\circ} \mathrm{C}$ was observed in DSC thermogram of the both $\mathrm{MCPBz}$ nanofibers attributed to ring opening reaction and cross- linking. Since these nanofibers have such low melting points and their curing temperatures are very high (above $200{ }^{\circ} \mathrm{C}$ ), photocuring was performed as a first step. Interestingly, after UV irradiation, melting transition peak was not observed in DSC thermograms of both DHBP-ad6 and DHBP-ad12 nanofibers verifying the enhancement of the thermal stability probably owing to the crosslinking provided by photo curing (Fig. 2, a, b). In addition, SEM images of DHBP-ad6 and DHBP-ad12 nanofibers show that UV irradiation did not deteriorate the fiber morphology (Fig. 3, c, g). Therefore, subsequently thermal curing was performed as a second step for UV-irradiated nanofibers at step-wise from $150{ }^{\circ} \mathrm{C}$ to $225^{\circ} \mathrm{C}$ to provide almost complete ring opening and cross-linking of the benzoxazine. Although directly thermal-cured $\mathrm{MCPBz}$ nanofibers lost the fibrous structure even at $150{ }^{\circ} \mathrm{C}$, UV-irradiated nanofibers perfectly preserved the fiber morphology during the thermal treatment which confirms the cross-linking achieved by two step curing without deteriorating the fiber morphology (Fig. 3, d, h).

In order to investigate the cross-linking of DHBP-ad6 and DHBPad12 nanofibers more detailed, the chemical structural changes occurring during the curing process were examined by FTIR spectroscopy, since characteristic benzoxazine peaks that observed at $920 \mathrm{~cm}^{-1}$ (benzene ring mode that is attached to the oxazine ring) and $1230 \mathrm{~cm}^{-1}$ (aromatic ether stretching of $\mathrm{C}-\mathrm{O}-\mathrm{C}$ ) and $1496 \mathrm{~cm}^{-1}$ (three substituted benzene ring mode) disappear with the ring opening reactions [1]. Fig. 4 represents the FTIR spectra of DHBP-ad6 and DHBP-ad12 nanofibers, after photo-curing with $1 \mathrm{~h}$ UV irradiation and after additional step-wise thermal curing of UVirradiated samples at $150{ }^{\circ} \mathrm{C}, 175^{\circ} \mathrm{C}, 200{ }^{\circ} \mathrm{C}$ and $225^{\circ} \mathrm{C}(1 \mathrm{~h}$ at each step). For both of the MCPBz nanofibers, characteristic benzoxazine peaks were appeared at same wavenumbers (920, 1230 and $1496 \mathrm{~cm}^{-1}$ ) since they have very similar chemical structure. After UV irradiation, characteristic absorbance bands were still observed in the FTIR spectra suggesting the ring opening and complete crosslinking were not achieved by photo-curing. However, intensity of the characteristic benzoxazine rings peaks in the FTIR spectra decreased and almost disappeared with the increasing temperature confirming the ring-opening and cross-linking through thermal curing.

Besides the SEM images and the FTIR spectroscopy results, physical properties of the MCPBz nanofibers after photo and thermal curing also confirm the cross-linking. First of all, the clear color change was observed during the curing process. As it is observed from the photographs in Fig. 5, white color of the as-electrospun nanofibers transformed to yellow with photo curing and then, yellow color of the UV-irradiated nanofibers transformed to
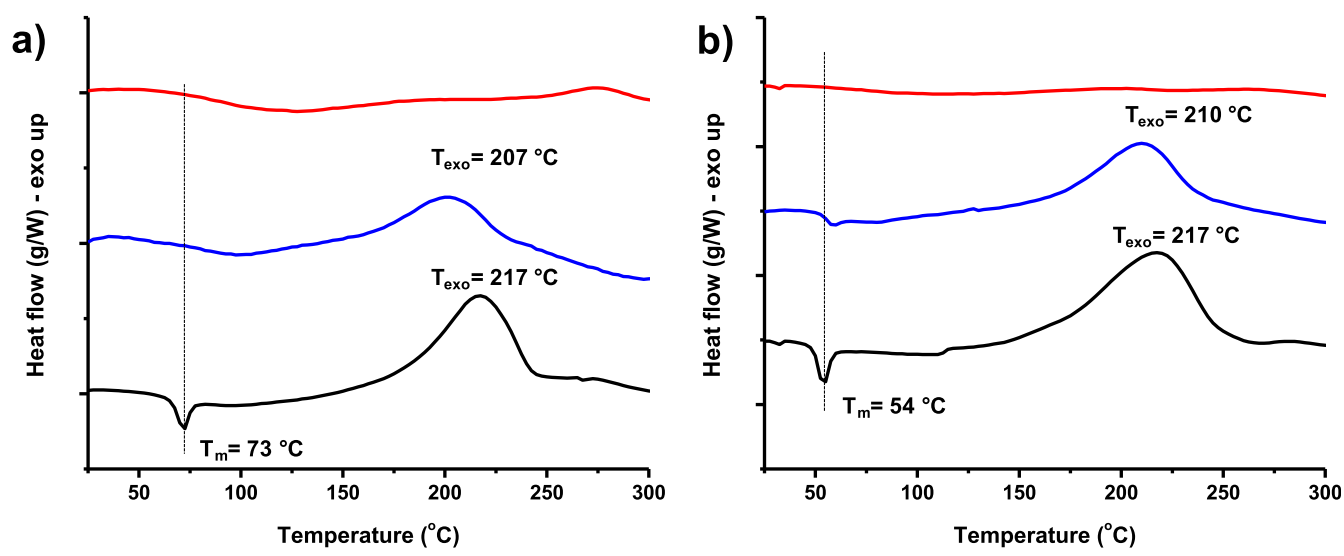

As-electrospun Photo-cured

Photo and thermal-cured

Fig. 2. DSC thermograms of a) DHBP-ad6 and b) DHBP-ad12 nanofibers. 

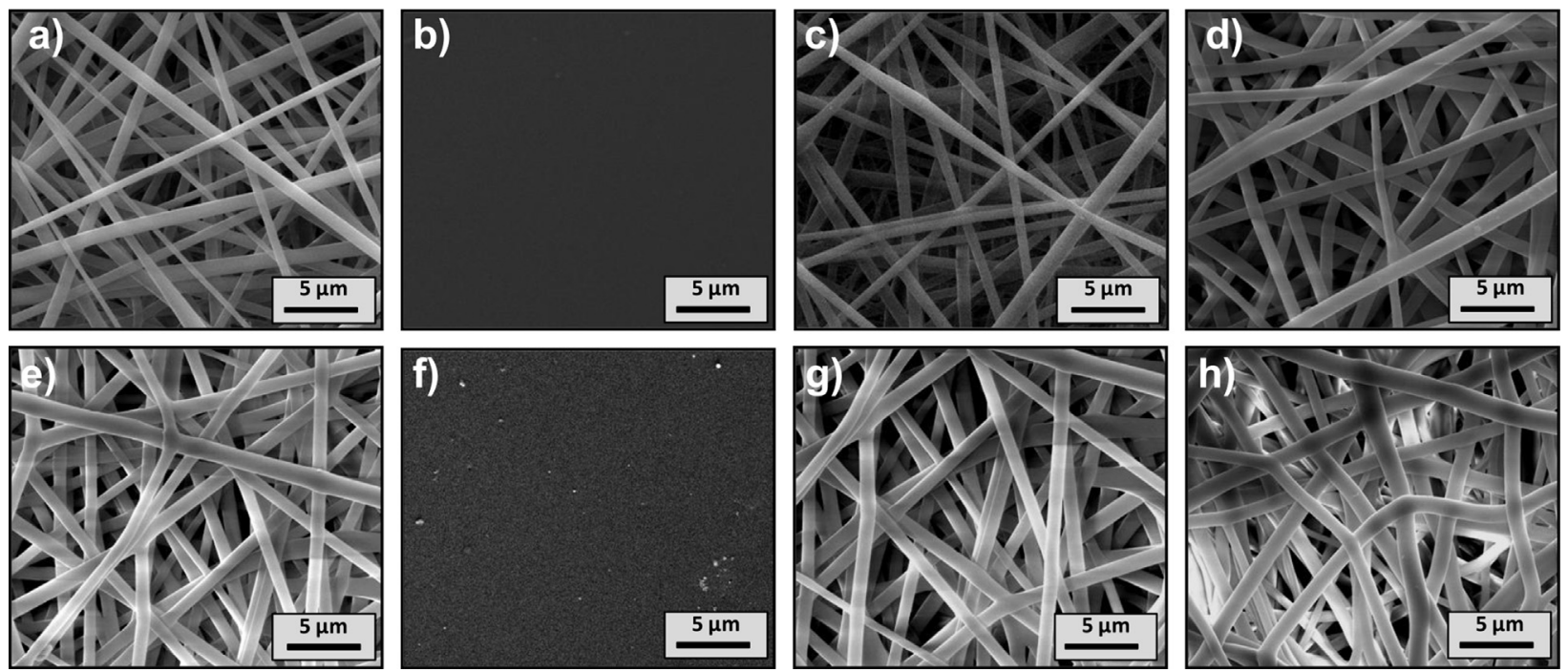

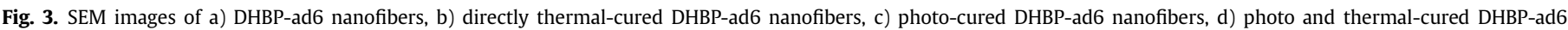

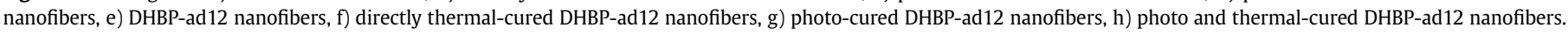

a)

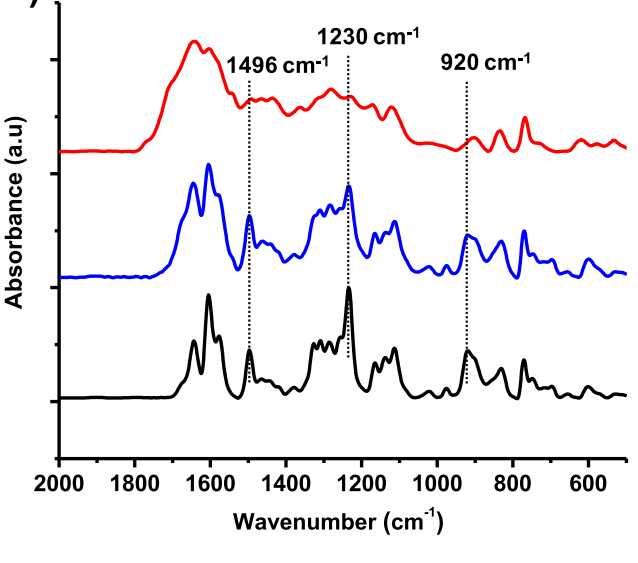

b)

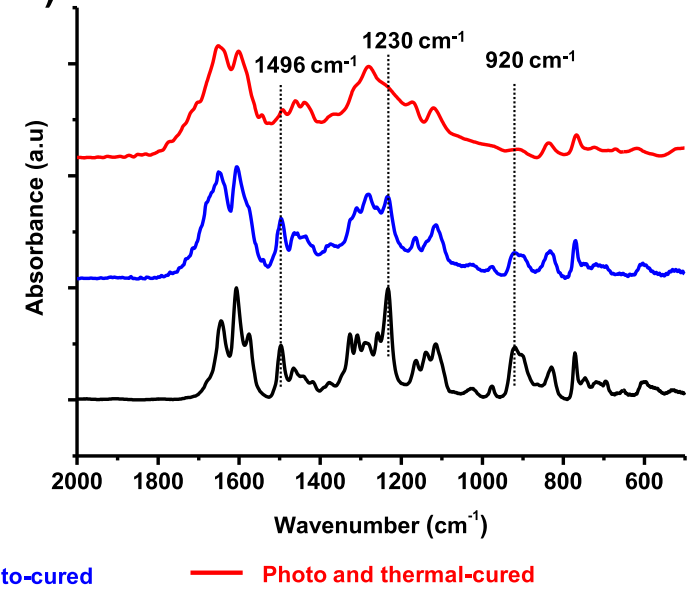

Fig. 4. FTIR spectra of a) DHBP-ad6 and b) DHBP-ad12 nanofibers.

brownish yellow which is a typical color of benzoxazine resins when they are thermally cured. Secondly, as-electrospun DHBP-ad6 nanofibers were very brittle, yet, after two-step curing they gained mechanical integrity and became more flexible. Although, both of the nanofibrous mats were obtained as free standing material, DHBP-ad12 nanofibrous mat was more flexible than the DHBP-ad6 nanofibrous mat presumably owing to the longer aliphatic chain structure of the DHBP-ad12 resins and they maintained the flexibility even after the two-step curing (Fig. 5).

In addition to the bending ability, mechanical properties of the photo and thermal-cured DHBP-ad6 and DHBP-ad12 nanofibrous mat were investigated. Stress-strain curves of the both cross-linked MCPBz nanofibrous mats obtained by DMA are given in Fig. 6. Three samples were used from each type of $\mathrm{MCPBz}$ nanofibrous mats for mechanical test and the results are summarized in Table 1. After photo and thermal curing, DHBP-ad6 and DHBP-ad12 nanofibrous mats showed significantly high Young's modulus compared to the directly thermal-cured MCPBz nanofibrous mats reported in the literature [29]. Young's modulus of DHBP-ad6 and DHBP-ad12 nanofibers were calculated as $2070 \pm 243$ and $264 \pm 59.66 \mathrm{MPa}$, respectively and this difference is expected since this value is highly dependent on the chain length. As the flexible aliphatic chain length decreases, stiffness of the polymeric material increases [39], hence, cross-linked DHBP-ad6 nanofibers showed remarkably higher Young's modulus then the cross-linked DHBP-ad12 nanofibers. In addition, ultimate tensile stress of the cross-linked DHBPad6 and DHBP-ad12 nanofibers were measured as $22.53 \pm 2.04$ and $15.29 \pm 2.48 \mathrm{MPa}$, respectively and these values are also fairly high from the reported work [29]. Here again not surprisingly the effect of the aliphatic chain length on the yield stress of the MCPBz nanofibrous mats was observed and cross-linked DHBP-ad6 nanofibrous mat having shorter aliphatic chain exhibited higher stress at yield which is very similar trend that was observed for the previously reported cross-linked polybenzoxazines [39]. Besides these, cross-linked DHBP-ad12 nanofibrous mat showed remarkably higher strain at break $(12.04 \pm 0.09)$ compared to the crosslinked DHBP-ad6 nanofibrous mat $(1.83 \pm 0.15)$ since as the aliphatic chain length increases, strain at break increases as well and these results consistent with the reported data [40].

The thermal properties of DHBP-ad6 and DHBP-ad12 nanofibers 

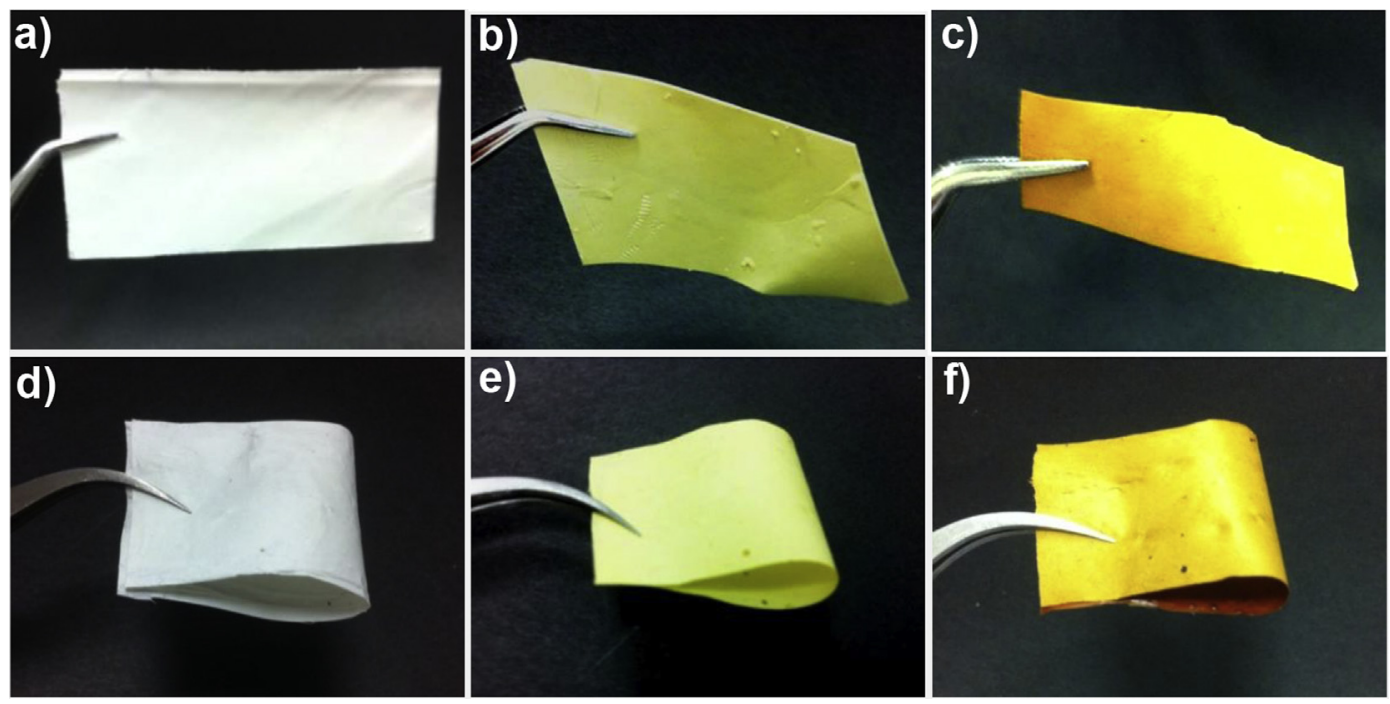

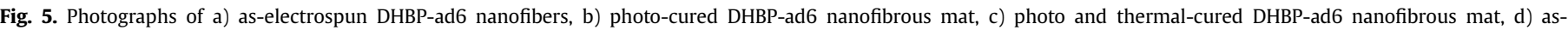
electrospun DHBP-ad12 nanofibrous mat, e) photo-cured DHBP-ad12 nanofibrous mat and f) photo and thermal-cured DHBP-ad12 nanofibers.

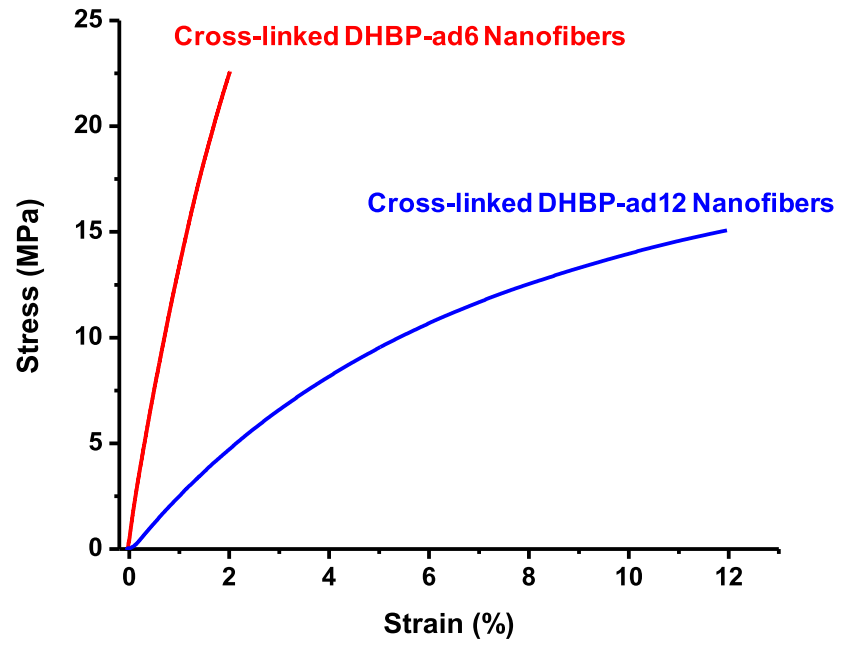

Fig. 6. Representative stress-strain curve of the photo and thermal-cured DHBP-ad6 and DHBP-ad12 nanofibrous mats.

were studied by TGA. After photo-curing, no significant change was observed in degradation temperatures (Td) of the nanofibers. On the other hand, thermal stability of the nanofibers was increased significantly after thermal curing (Fig. S4). Td onset and $\mathrm{Td}$ maximum values of the DHBP-ad6 nanofibers were observed at $231{ }^{\circ} \mathrm{C}$ and $450{ }^{\circ} \mathrm{C}$ after photo curing. On the other hand, an increase was observed in $\mathrm{Td}$ onset and $\mathrm{Td}$ maximum values of the DHBP-ad6 nanofibers after thermal curing which was measured as $250{ }^{\circ} \mathrm{C}$ and $458^{\circ} \mathrm{C}$, respectively. In addition char yield of the DHBPad6 nanofibers increased from $41 \%$ to $53 \%$ after photo and thermal curing. Likewise, thermal properties of DHBP-ad12 nanofibers showed the same trend each step of curing. Td onset, Td maximum and char yield were measured as $245{ }^{\circ} \mathrm{C}, 474{ }^{\circ} \mathrm{C}$ and $18.4 \%$, respectively. There was no significant change in these values of the DHBP-ad12 nanofibers after photo curing $\left(242{ }^{\circ} \mathrm{C}, 473{ }^{\circ} \mathrm{C}\right.$ and $20.3 \%$ ); however, noticeable increase was observed especially at $\mathrm{Td}$ onset and char yield of these materials after thermal curing $\left(270{ }^{\circ} \mathrm{C}\right.$, $476{ }^{\circ} \mathrm{C}$ and $35.1 \%$ ) (Fig. S4 and Table S2). These findings indicate that through two-step curing, as the chain length increases, the thermal stability of $\mathrm{MCPBz}$ nanofibers increases whereas the char yield of the MCPBz nanofibers decreases as reported earlier work [39].

\subsection{Solubility and stability test of cross-linked DHBP-ad6 and DHBP-ad12 nanofibers}

Cross-linking of the photo and thermal-cured nanofibers were further investigated by solubility and stability tests. In this part, photo and thermal-cured nanofibers were immersed for $24 \mathrm{~h}$ into different solvents such as chloroform, DMF, 1,4-dioxane, DMAc and THF which are very good solvents for the DHBP-ad6 and DHBPad12 resins. Fig. 7 represents the SEM images of photo and thermal-cured DHBP-ad6 and DHBP-ad12 nanofibers after the solubility test in chloroform and DMF. It is clearly observed that photo and thermal-cured DHBP-ad6 and DHBP-ad12 nanofibers were not dissolved in these solvents in which the homogenous electrospinning solutions were prepared to produce bead free and uniform DHBP-ad6 and DHBP-ad12 nanofibers. Although, photocured DHBP-ad12 nanofibers have shown negligible deformation with lower mechanical integrity after immersing chloroform and DMF, photo-cured DHBP-ad6 nanofibers maintained the fibrous structure (Fig. 7, a1, a2, c1, c2). On the other hand, after thermal curing, both DHBP-ad6 and DHBP-ad12 nanofibers have gained better mechanical integrity and they preserved the original fiber morphology (Fig. 7, b1, b2, d1, d2). Same behavior of photo and thermal-cured DHBP-ad6 and DHBP-ad12 nanofibers were observed in 1,4-dioxane, DMAc and THF as well (Fig. S5).

Table 1

Mechanical properties of the DHBP-ad6 and DHBP-ad12 nanofibrous mat after photo and thermal curing.

\begin{tabular}{|c|c|c|c|}
\hline Nanofibrous mat & Stress at yield (MPa) & Strain at break (\%) & Young's modulus (MPa) \\
\hline DHBP-ad6 & $22.53 \pm 2.04$ & $1.83 \pm 0.15$ & $2070 \pm 243$ \\
\hline DHBP-ad12 & $15.29 \pm 2.48$ & $12.04 \pm 0.09$ & $264 \pm 59.66$ \\
\hline
\end{tabular}



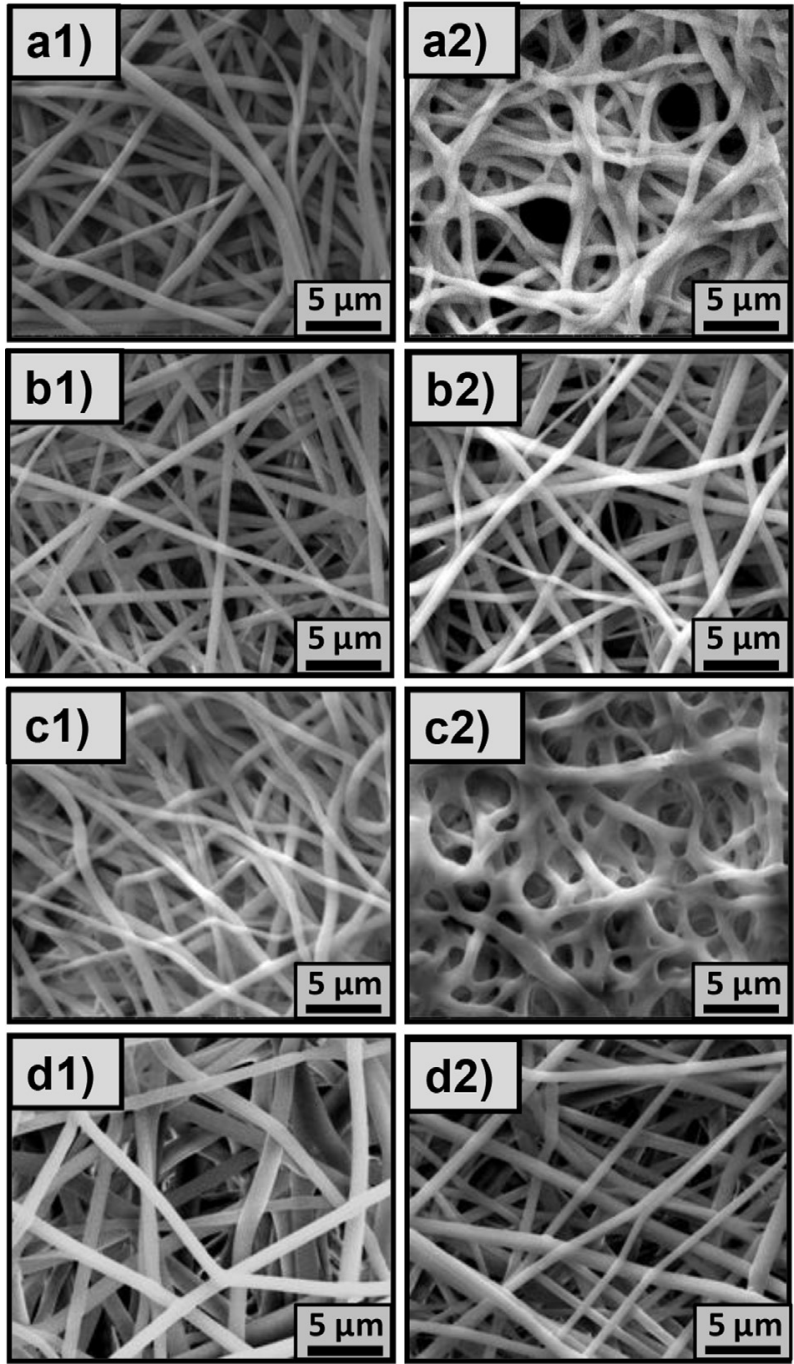

Fig. 7. SEM images of cross-linked nanofibers after immersing $24 \mathrm{~h}$ in 1) chloroform and 2) DMF; a) photo-cured DHBP-ad6 nanofibers, b) photo and thermal-cured DHBPad6 nanofibers, $\mathrm{c}$ ) photo-cured DHBP-ad12 nanofibers and d) photo and thermal-cured DHBP-ad12 nanofibers.
In addition, strong acids were used to test the stability of crosslinked nanofibers in harsh conditions. High concentration solutions ( $5 \mathrm{M}$ ) of $\mathrm{HCl}, \mathrm{HNO}_{3}$ and $\mathrm{H}_{2} \mathrm{SO}_{4}$ were prepared to demonstrate the stability of DHBP-ad6 and DHBP-ad12 nanofibers. Since ring opening and complete cross-linking was not provided by photocuring, fibrous structure of DHBP-ad6 was deteriorated in $\mathrm{HCl}$ and lost completely in $\mathrm{HNO}_{3}$ and $\mathrm{H}_{2} \mathrm{SO}_{4}$ (Fig. S6, a1-a3). In the case of photo-cured DHBP-ad12 nanofibers, the fibrous structure was maintained when treated with $\mathrm{HCl}$ and $\mathrm{HNO}_{3}$ whereas $\mathrm{H}_{2} \mathrm{SO}_{4}$ deformed the nanofibers (Fig. S6, b1-b3). On the other hand, structural integrity of the photo and thermal-cured DHBP-ad6 and DHBP-ad12 nanofibers were great, thus they were able to stay as free standing webs under strong acid treatments and the fibrous structure were preserved perfectly (Fig. 8).

Moreover, thermal stability of cross-linked DHBP-ad6 and DHBP-ad12 nanofibers were investigated within the temperature range $\left(250-400{ }^{\circ} \mathrm{C}\right)$ which is higher than the curing temperatures and the lower than the decomposition temperatures of the crosslinked $\mathrm{MCPBz}$ nanofibers. It was observed that DHBP-ad6 and DHBP-ad12 nanofibers are structurally stable even at very high temperatures $\left(400{ }^{\circ} \mathrm{C}\right.$ ) demonstrating that they are highly crosslinked thermoset materials having nanofibrous morphology (Fig. S7).

\section{Conclusion}

This paper demonstrates the feasibility of the cross-linking of polybenzoxazine based electrospun nanofibers obtained from the main-chain polybenzoxazines ( $\mathrm{MCPBz}$ ) with long aliphatic chain by two-step curing. The structure of the MCPBz were tailored to be able to crosslink through UV-light initiated free radical polymerization and thermally induced ring-opening reactions, and as a result DHBP-ad6 and DHBP-ad12 resins with different aliphatic diamine chain length were synthesized. Bead-free and uniform nanofibers were obtained from both DHBP-ad6 and DHBP-ad12 resins when the solution concentrations were 35\% (w/v) and $25 \%$ $(\mathrm{w} / \mathrm{v})$, respectively. DSC measurements indicated that melting transition peak of as-electrospun $\mathrm{MCPBz}$ nanofibers were disappeared after photo curing illustrating the enhancement of the thermal stability of MCPBz nanofibers. In addition, SEM images of the photo and thermal-cured nanofibers show that the fiber morphology has preserved after each step of curing. Structural
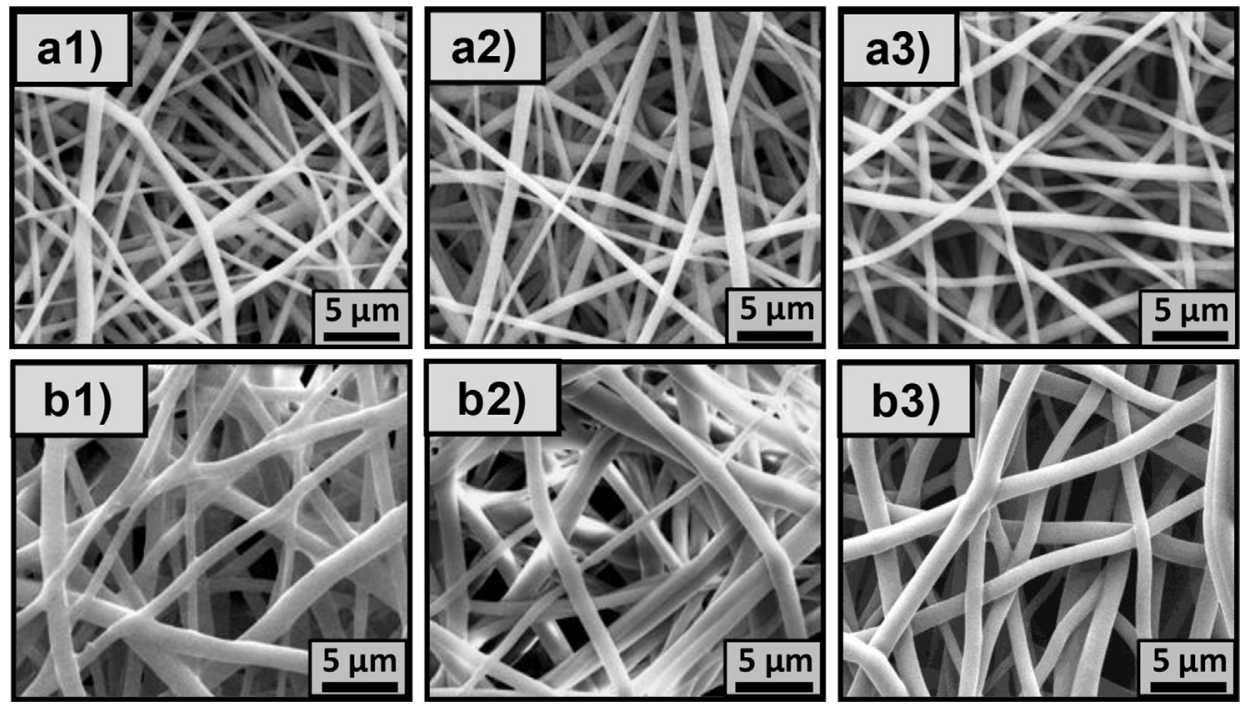

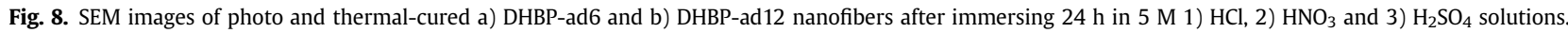


changes occurring during the photo and thermal curing were investigated by FTIR spectroscopy. Although characteristic absorbance bands of the benzoxazine structure were observed after photo curing, those bands were disappeared during the thermal curing verifying the achievement of ring-opening and cross-linking only by thermal curing. Moreover, TGA thermograms of DHBP-ad6 and DHBP-ad12 nanofibers show that the Td onset and char yield of the MCPBz nanofibers have increased only through thermal curing. Furthermore, DMA analysis illustrated that cross-linked DHBP-ad6 and DHBP-ad12 nanofibrous web have quite high Young's modulus $(2070 \pm 243$ and $264 \pm 59.66 \mathrm{MPa}$, respectively) and tensile stress $(22.53 \pm 2.04$ and $15.29 \pm 2.48 \mathrm{MPa}$, respectively) compared to the previously reported $\mathrm{MCPBz}$ nanofibrous web [29]. Also, tensile strain of the cross-linked DHBP-ad12 nanofibers was significantly higher than the cross-linked DHBP-ad6 nanofibers suggesting the longer aliphatic chain length probably has increased the flexibility of nanofibers resulted in higher elongation at break. Besides, solubility and stability experiments demonstrated that these nanofibers preserved the fibrous structure and mechanical integrity in good solvents (chloroform, DMF, 1,4-dioxane, DMAc and THF), highly concentrated $(5 \mathrm{M})$ strong acids $\left(\mathrm{HCl}, \mathrm{HNO}_{3}, \mathrm{H}_{2} \mathrm{SO}_{4}\right)$ and at high temperatures $\left(400{ }^{\circ} \mathrm{C}\right.$ ). In brief, we obtained highly crosslinked thermoset nanofibrous polybenzoxazine-based materials with good mechanical and thermal properties and highly stable in organic solvents and harsh acidic conditions, and these properties may be quite useful for the certain applications requiring high temperatures and harsh acidic conditions or organic solvents.

\section{Acknowledgments}

Dr. T. Uyar acknowledges The Turkish Academy of Sciences Outstanding Young Scientists Award Program (TUBA-GEBIP) for partial funding. Y. Ertas acknowledges TUBITAK (2211-C) for the PhD student scholarship.

\section{Appendix A. Supplementary data}

Supplementary data related to this article can be found at http:// dx.doi.org/10.1016/j.polymer.2015.12.026.

\section{References}

[1] H. Ishida, T. Agag, Handbook of Benzoxazine Resins, Elsevier, 2011.

[2] S. Ramakrishna, K. Fujihara, W.-E. Teo, T.-C. Lim, Z. Ma, An Introduction to
Electrospinning and Nanofibers, World Scientific, 2005.

[3] J.H. Wendorff, S. Agarwal, A. Greiner, Electrospinning: Materials, Processing, and Applications, John Wiley \& Sons, 2012.

[4] J. Sun, W. Wei, Y. Xu, J. Qu, X. Liu, T. Endo, RSC Adv. 5 (2015) 19048-19057.

[5] C. Li, Q. Ran, R. Zhu, Y. Gu, RSC Adv. 5 (2015) 22593-22600.

[6] P. Yang, Y. Gu, J. Polym. Sci. Pol. Chem. 50 (2012) 1261-1271.

[7] H. Zhang, W. Gu, R. Zhu, Q. Ran, Y. Gu, Polym. Degrad. Stabil. 111 (2015) $38-45$.

[8] P. Yang, X. Wang, H. Fan, Y. Gu, Phys. Chem. Chem. Phys, 15 (2013) $15333-15338$.

[9] W.-K. Kim, W.L. Mattice, Langmuir 14 (1998) 6588-6593.

[10] C.H. Lin, Y.S. Shih, M.W. Wang, C.Y. Tseng, T.Y. Juang, C.F. Wang, RSC Adv. 4 (2014) 8692-8698.

[11] B. Kiskan, Y. Yagci, J. Polym. Sci. Pol. Chem. 52 (2014) 2911-2918.

[12] J. Shi, X. Zheng, L. Xie, F. Cao, Y. Wu, W. Liu, Eur. Polym. J. 49 (2013) 4054-4061.

[13] J. Liu, X. Lu, Z. Xin, C. Zhou, Langmuir 29 (2012) 411-416.

[14] D. Baranek, L.L. Kendrick, J. Narayanan, G.E. Tyson, S. Wand, D.L. Patton, Polym. Chem. 3 (2012) 2892-2900.

[15] H. Lin, S.L. Chang, T.Y. Shen, Y.S. Shih, H.T. Lin, C.F. Wang, Polym. Chem. 3 (2012) 935-945.

[16] L. Qu, Z. Xin, Langmuir 27 (2011) 8365-8370.

[17] M. Nakamura, H. Ishida, Polymer 50 (2009) 2688-2695.

[18] B. Kiskan, Y. Yagci, H. Ishida, J. Polym. Sci. Pol. Chem. 46 (2008) 414-420.

[19] T. Takeichi, T. Kano, T. Agag, Polymer 46 (2005) 12172-12180.

[20] P. Lorjai, S. Wongkasemjit, T. Chaisuwan, A.M. Jamieson, Polym. Degrad. Stab. 96 (2011) 708-718.

[21] S. Mahadik-Khanolkar, S. Donthula, A. Bang, C. Wisner, C. Sotiriou-Leventis, N. Leventis, Chem. Mater 26 (2014) 1318-1331.

[22] D.A. Rubenstein, H. Lu, S.S. Mahadik, N. Leventis, W. Yin, J. Biomater. Sci. Polym. E. 23 (2012) 1171-1184.

[23] T. Chaisuwan, T. Komalwanich, S. Luangsukrerk, S. Wongkasemjit, Desalination 256 (2010) 108-114.

[24] P. Katanyoota, T. Chaisuwan, A. Wongchaisuwat, S. Wongkasemjit, Mater. Sci. Eng. B Solid 167 (2010) 36-42.

[25] P. Lorjai, T. Chaisuwan, S. Wongkasemjit, J. Sol-gel Sci. Technol. 52 (2009) 56-64.

[26] O.S. Taskin, B. Kiskan, A. Aksu, N. Balkis, J. Weber, Y. Yagci, Chem A Eur. J. 20 (2014) 10953-10958.

[27] K. Pakkethati, N. Tungsattabutrat, T. Chaisuwan, S. Wongkasemjit, Mater. Res. Innov, 16 (2012) 243-248.

[28] Y. Ertas, T. Uyar, Polymer 55 (2014) 556-564.

[29] H.Y. Li, Y.Y. Lee, Y. Chang, C.H. Lin, Y.L. Liu, Adv. Mater. Inter 2 (2015) 1500065.

[30] F. Kasapoglu, I. Cianga, Y. Yagci, T. Takeichi, J. Polym. Sci. Pol. Chem. 41 (2003) 3320-3328.

[31] Y. Yagci, Macromol. Symp. 240 (2006) 93-101.

[32] J. Bai, Z. Shi, J. Yin, Polymer 54 (2013) 2498-2505.

[33] G. Temel, D.S. Esen, N. Arsu, Polym. Eng. Sci. 52 (2012) 133-138.

[34] J. Bai, Z. Shi, J. Appl. Polym. Sci. 128 (2013) 1785-1791.

[35] J. Shi, P. Chen, K. Wang, J. Lu, J. Nie, Polym. Sci. Ser. B 56 (2014) 632-638.

[36] L. Jin, T. Agag, Y. Yagci, H. Ishida, Macromolecules 44 (2011) 767-772.

[37] R. Liska, J. Polym, Sci. Polym. Chem. 40 (2002) 1504-1518.

[38] T. Uyar, F. Besenbacher, Polymer 49 (2008) 5336-5343.

[39] D.J. Allen, H. Ishida, J. Appl. Polym. Sci. 101 (2006) 2798-2809.

[40] A.D. Baranek, L.L. Kendrick, J. Narayanan, G.E. Tyson, S. Wand, D.L. Patton, Polym. Chem. 3 (2012) 2892-2900. 